\title{
Unique genetic loci identified for emotional behavior in control and chronic stress conditions
}

\author{
Kimberly A. K. Carhuatanta ${ }^{1,2}$, Chloe J. A. Shea ${ }^{1}$, James P. Herman ${ }^{3}$ and Ryan Jankord ${ }^{1 *}$ \\ ${ }^{1}$ Applied Neuroscience, 711th Human Performance Wing, Air Force Research Laboratory, Wright-Patterson Air Force Base, OH, USA \\ ${ }^{2}$ Research Associate Program, National Research Council, National Academies of Sciences, Washington, DC, USA \\ ${ }^{3}$ Department of Psychiatry and Behavioral Neuroscience, University of Cincinnati, Cincinnati, OH, USA
}

\section{Edited by:}

Mathias V. Schmidt, Max Planck Institute of Psychiatry, Germany

Reviewed by:

Michael F. Miles, Virginia

Commonwealth University, USA

Alberto Fernández-Teruel,

Autonomous University of

Barcelona, Spain

${ }^{*}$ Correspondence:

Ryan Jankord, Applied

Neuroscience, 711th Human

Performance Wing, Air Force

Research Laboratory, Bldg. 840,

2510 Fifth St., Wright-Patterson Air

Force Base, $\mathrm{OH} 45433$, USA

e-mail: ryan.jankord@us.af.mil
An individual's genetic background affects their emotional behavior and response to stress. Although studies have been conducted to identify genetic predictors for emotional behavior or stress response, it remains unknown how prior stress history alters the interaction between an individual's genome and their emotional behavior. Therefore, the purpose of this study is to identify chromosomal regions that affect emotional behavior and are sensitive to stress exposure. We utilized the BXD behavioral genetics mouse model to identify chromosomal regions that predict fear learning and emotional behavior following exposure to a control or chronic stress environment. 62 BXD recombinant inbred strains and C57BL/6 and DBA/2 parental strains underwent behavioral testing including a classical fear conditioning paradigm and the elevated plus maze. Distinct quantitative trait loci (QTLs) were identified for emotional learning, anxiety and locomotion in control and chronic stress populations. Candidate genes, including those with already known functions in learning and stress were found to reside within the identified QTLs. Our data suggest that chronic stress history reveals novel genetic predictors of emotional behavior.

Keywords: BXD, QTL, stress, fear learning, anxiety, genetics, emotional behavior

\section{INTRODUCTION}

Much of an individual's emotional behavior and response to stress is determined by genetic factors. Mood and anxiety disorders are highly heritable (Bienvenu et al., 2011; Binder, 2012). Environmental stressors often exacerbate psychiatric disease in individuals diagnosed with mood and anxiety disorders, resulting in attention deficits, difficulty in memory tasks, irritability, and affect changes in humans (Renoir et al., 2013). In rodents, chronic stress, and hypothalamus-pituitary- adrenal axis (HPA) activation result in anhedonia, sleep abnormalities, circadian disturbances, and memory impairment (Herman and Watson, 1995; Pardon et al., 2000a,b; de Kloet et al., 2005). Additionally, emotional learning, and anxiety/exploratory behavior are subject to stress-induced effects (Mozhui et al., 2010).

While the effects of both genetics and environment on emotional behavior have been described (Valentinuzzi et al., 1998; Mozhui et al., 2010), to date, the interplay between all three (i.e., genetics, environment, and emotional behavior) has been less studied. In humans, the interaction of genetic factors and chronic stress has been investigated in populations having high stress lifestyles or occupations such as medical or nursing residents and military or fire-fighting service positions or low socioeconomic status (Reijneveld and Schene, 1998; Chernomas and Shapiro, 2013; Powell et al., 2013). For in depth genetic analysis of behavior, researchers can rely on recombinant inbred strains, such as the BXD mouse strain populations (Philip et al., 2010). The BXD mouse population is a behavioral genetics model of recombinant inbred mouse strains derived from the C57BL/6 and $\mathrm{DBA} / 2$ parental strains, and is used by researchers to map phenotypic variation across RI BXD strains onto defined chromosomal regions via quantitative trait loci (QTL) analyses (Peirce et al., 2004; Andreux et al., 2012). Based on the differences in performance and stress responsiveness between C57BL/6 and DBA/2 strains (Waddell et al., 2004; Brigman et al., 2009; Mozhui et al., 2010), the BXD mouse population provides a unique opportunity to identify the chromosomal locations that contribute to emotional behavior.

Here, we test the hypothesis that environmental context (the presence or absence of stress) reveals unique genetic predictors of emotional behavior. Emotional behavior of 62 BXD strains, and C57BL/6 and DBA/2 parental strains was assessed by fear conditioning and performance on the elevated plus maze. Littermates of each strain were treated with either control or chronic variable stress (CVS). QTL mapping was performed to identify genetic loci and candidate genes underlying emotional learning and behavior in both the control and chronic stress states.

\section{MATERIALS AND METHODS ETHICS STATEMENT}

All animal procedures were approved by the Air Force Research Laboratory Institutional Animal Care and Use Committee in accordance with the NIH Guide for the Care and Use of Laboratory Animals.

\section{ANIMALS}

All BXD recombinant inbred strains and their parental C57BJ/6 and $\mathrm{DBA} / 2$ strains were purchased in littermate pairs from Jackson Laboratory (Bar Harbor, ME, USA). Male mice, aged 9 
weeks at start of experiments, were singly housed with ad libitum access to standard rodent chow (Harlan Teklad, Madison, WI, USA) and water. Ambient housing conditions were controlled for temperature $\left(18-24^{\circ} \mathrm{C}, 21^{\circ} \mathrm{C}\right.$ average), humidity $(30-70 \%, 35 \%$ average), and the housing room maintained a standard 12:12 light cycle (6 a.m. on). Sixty-two different BXD strains (592 BXD mice, $n=3-5$ per strain per group), in addition to the C57BJ/ 6 and $\mathrm{DBA} / 2$ parental strains ( $n=5$ per strain per group), were utilized in the study.

\section{EXPERIMENTAL DESIGN}

The design of this study is summarized in Figure 1. Upon arrival at the animal facility, littermate pairs were divided into control and chronic stress (CVS) populations and were allowed to acclimate to our animal facility and to single housing for a 10 days period. Both populations of animals were housed in the same room. All treatment and testing occurred during Monday-Friday of the week during the light cycle. After 2 days of handling, the control population began behavioral testing (weeks 1-3) and the CVS population began the chronic stress paradigm (weeks 1-4). The CVS paradigm lasted 4 weeks with behavioral testing being completed during weeks 2-4 for CVS mice. Behavioral testing in the chronic stress population was offset for 1 week from environmental controls to allow 1 week of exposure to the CVS paradigm before behavioral testing and to accommodate throughput limitations of the behavioral assays. All mice underwent 3 weeks of behavioral testing such that the elevated plus maze (EPM) task preceded the learning tasks. Following the EPM, mice complete a 7 days Morris water maze (data not described in this manuscript), followed by the fear conditioning test. The order of tests permitted the observation of anxiety-like behavior prior to any conditioning effects. Following the completion of all behavioral tests, animals were euthanized via rapid decapitation.

Ten cohorts (50-64 mice) of BXD strains (25-32 strains per cohort) were utilized over the span of a year. Each cohort consisted of two littermates of each strain and had a unique grouping of BXD strains to minimize confounding factors such as seasonal and group effects.

\section{CHRONIC STRESS TREATMENT}

Chronic stress was established using the CVS model (Herman and Watson, 1995; Furay et al., 2008; Castañeda et al., 2011). In this paradigm, animals underwent a randomized schedule of one to two mild to moderate physiological and psychological stressors daily 5 days a week. Stressors included novel overnight home cage, hypoxia $(30 \mathrm{~min})$, cold exposure $\left(15 \mathrm{~min}\right.$ at $\left.4^{\circ} \mathrm{C}\right)$, open field (30 $\mathrm{min})$, and constant motion (60 $\mathrm{min}$ ) exposure. The randomization of the schedule resulted in unique order and sequence of the stressors thereby limiting predictability and habituation to the chronic stress paradigm. All cohorts of animals were exposed to the same order and sequence of stressors.

\section{ELEVATED PLUS MAZE (EPM)}

Prior to any additional behavioral testing, mice were subjected to the EPM in which they were placed in the center of the EPM apparatus, and recorded and monitored using EthoVision XT software (Noldus Information Technologies, Leesburg, VT) for 5 min. Experimenter did not remain in EPM testing area during data collection. The EPM is elevated $1 \mathrm{~m}$ above the floor and consists of two open and closed arms $(40 \times 8 \mathrm{~cm})$ separated by a center area. Closed arms contain vertical gray plexiglass walls $(29.5 \mathrm{~cm})$, while open arms contain no protective edges. During testing, the maze is illuminated by two $60 \mathrm{~W}$ incandescent light bulbs placed approximately $35 \mathrm{~cm}$ above the arms in a room illuminated by two shielded $150 \mathrm{~W}$ incandescent light bulbs. The measures collected via EthoVisionXT software (Nolud Information Technologies, Leesburg, VT) from the EPM test include: (1) time spent in the closed arms, (2) time spent in the open arm, and (3) distance traversed throughout the maze. Time spent in the closed arms has been associated with anxietylike behavior, while time in open arms is considered exploratory. Distance traversed was assessed as a measure of locomotor activity within BXD strains.

\section{FEAR CONDITIONING PARADIGM}

Experiments were completed using four fear conditioning chambers (Med Associates, Inc., St. Albans, VT, USA). Chambers

\section{Study Design}

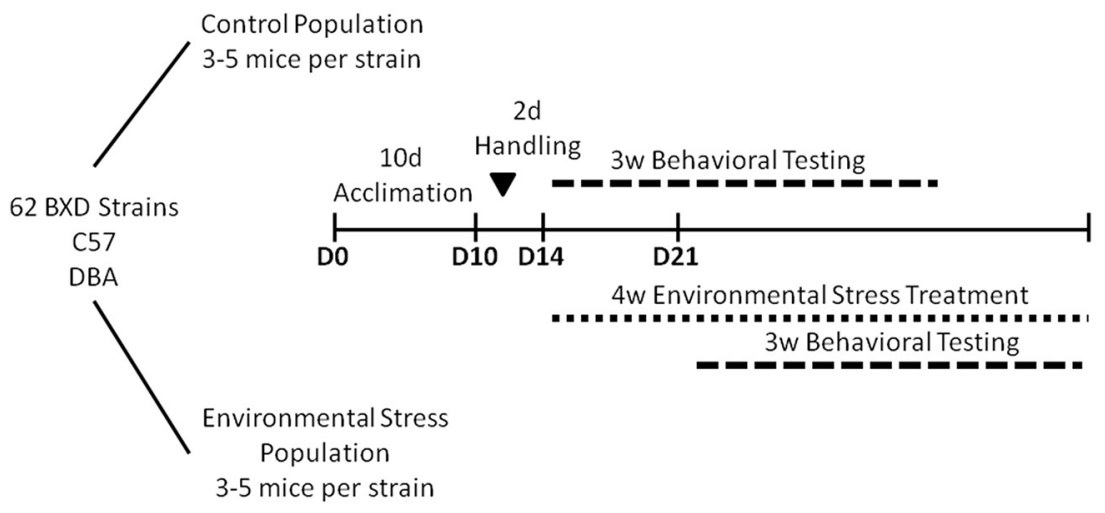

FIGURE 1 | Study Design. 


\section{A Freezing to Training}

Control

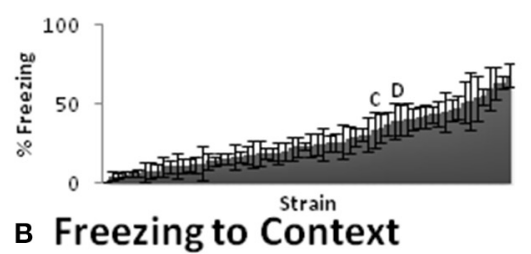

Control

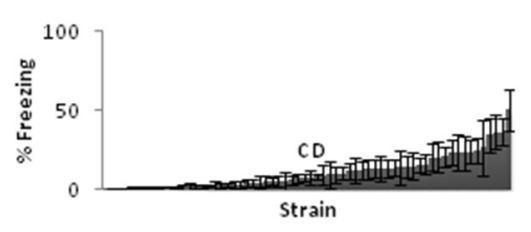

c Freezing to Tone

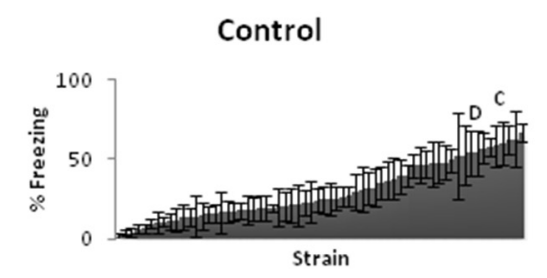

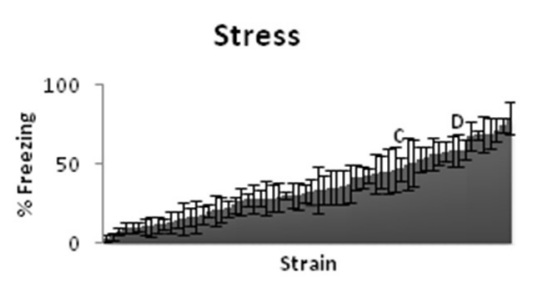

Stress

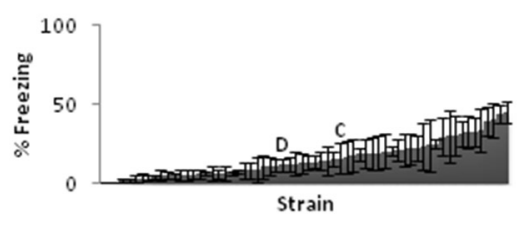

Stress

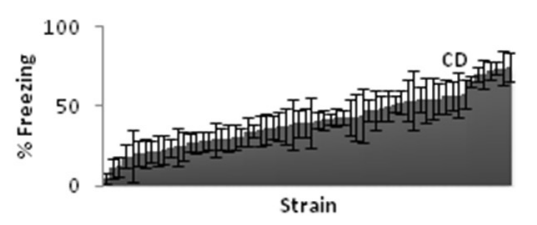

Stress Effect

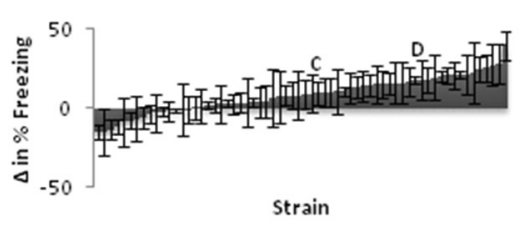

Stress Effect

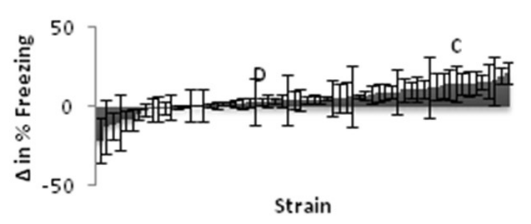

Strain

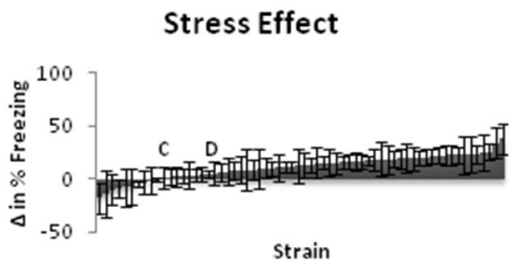

FIGURE 2 | Behavioral phenotypes of fear conditioning paradigm. Mean \pm s.e.m. \% time freezing for Control (Left) and Stress (Middle) populations, and mean \pm s.e.m. difference in \% freezing between littermates (Stress minus Control: Stress Effect, Right) of the (A) Training (Day 1), (B) Context (Day 2), (C) Tone (Day 3). C and $\mathbf{D}$ indicate the locations of parental strains C57BL/6 and DBA/2 strains among the rankings, respectively. consist of front and back Plexiglas and side metal walls. Fear conditioning chambers were housed within camera containing sound attenuation cubicles that reduce outside sound and light during testing. Fear Conditioning was completed over 3 days. On day 1 , mice were placed into the operant chambers (with steel grid floor) and freezing behavior is assessed using EthoVision XT software (Noldus Information Technologies, Leesburg, VT) throughout the session. Day 1 session included four exposures to a $30 \mathrm{~s}$ tone $(85 \mathrm{~dB}, 3 \mathrm{KHz})$ that terminated with a $2 \mathrm{~s}$ footshock $(0.75 \mathrm{~mA})$, which elicited freezing behavior (unconditioned response) that was captured via EthoVision software. Each tone was separated by a $30 \mathrm{~s}$ inter-tone interval. The pairing of the tone and shock (unconditioned stimulus, US) on Day 1 resulted in the establishment of the tone as a conditioned stimulus (CS), and elicited freezing behavior (conditioned response). On Day 2, mice were returned to the operant chamber and freezing behavior was monitored for $10 \mathrm{~min}$. On Day 3, the environmental context of the operant chamber was altered by replacing the grid floor with a smooth white Plexiglas insert. Mice were then exposed to the $30 \mathrm{~s}$ CS (tone) 9 times with random inter-tone intervals.

We measured the percent time displaying freezing behavior on Day 1 during acquisition (180 s following the final exposure to the CS-US pairing), Day 2 after placement within the conditioning context (180 s), and Day 3 during the presentation of the auditory cue $(30 \mathrm{~s})$.

\section{Statistics}

Stress-effect was calculated as the difference in performance between control and CVS littermates. Mixed model analysis was performed via lme 4 and lmerTest packages in $\mathrm{R}$ using stress, strain, month of testing, and experimenter handling during testing as fixed variables and cohort as a random variable. Non-linear mixed-effect test was followed by ANOVA to obtain values for main effects of fixed variables. Pearson product-moment correlations ( $\mathrm{R}$ ) and Spearman rank order correlations (rho) between traits measured were computed via GeneNetwork for both the Control and Stress population.

\section{Summary statistics of heritability of behavioral traits measured in FC and EPM testing}

Broad-sense $\left(H^{2}\right)$ and narrow-sense $\left(h^{2}\right)$ heritability of expression levels in the recombinant inbred lines was estimated using the Hegmann and Possidente Method (1981): $H^{2}=\mathrm{Va} / \mathrm{Vt} ; h^{2}=$ $1 / 2 \mathrm{Va} /(1 / 2 \mathrm{Va}+\mathrm{Vw})$. $\mathrm{Va}$ is the variance among strains, $\mathrm{Vt}$ is the variance in the total population, and $\mathrm{Vw}$ is the variance within strains.

\section{OTL MAPPING}

QTL mapping was performed using complex trait analysis and mapping tools available on the Genenetwork website (http:// www.genenetwork.org). GeneNetwork utilizes 3806 markers (intermarker interval of $0.66 \mathrm{Mb}$ ) in $89 \mathrm{BXD}$ recombinant inbred 


\section{A Locomotion}

Control

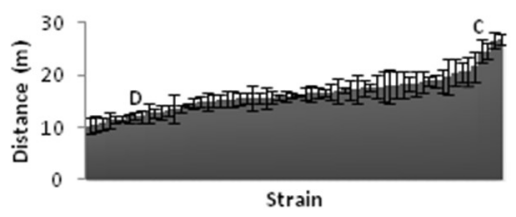

\section{B Time in Closed Arm}

Control

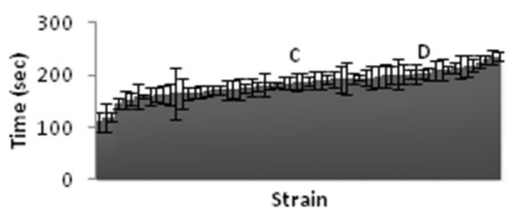

\section{c Time in Open Arm}

Control

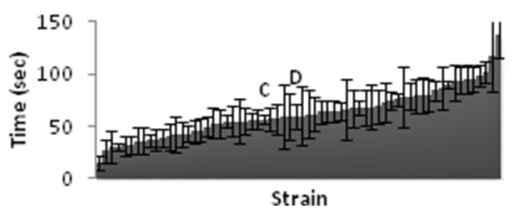

Stress

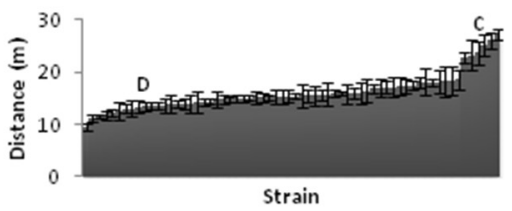

Stress

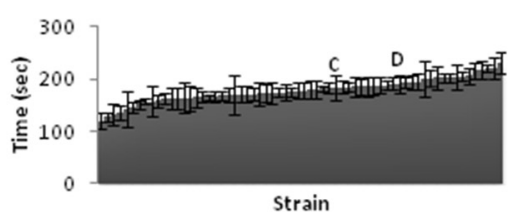

Stress

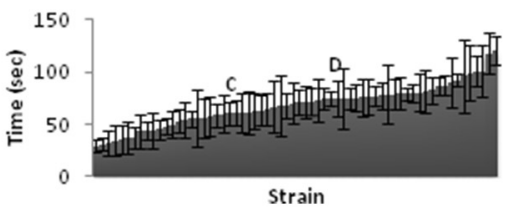

Stress Effect

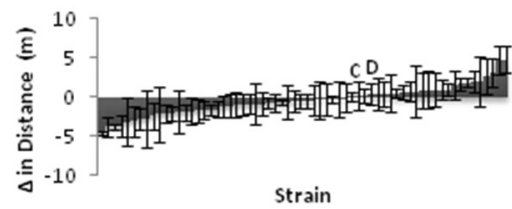

FIGURE 3 | Behavioral phenotypes of the elevated plus maze. Mean \pm s.e.m. distance or time spent for Control (Left) and Stress (Middle) populations, and mean \pm s.e.m. difference in distance or time spent between littermates
(Stress minus Control: Stress Effect, Right) of the (A) Locomotion, (B) Time in Closed Arm, and (C) Time in Open Arm. $\mathbf{C}$ and $\mathbf{D}$ indicate the locations of parental strains C57BL/6 and DBA/2 strains among the rankings, respectively.

Table 1 | Correlation of traits measured in control population.

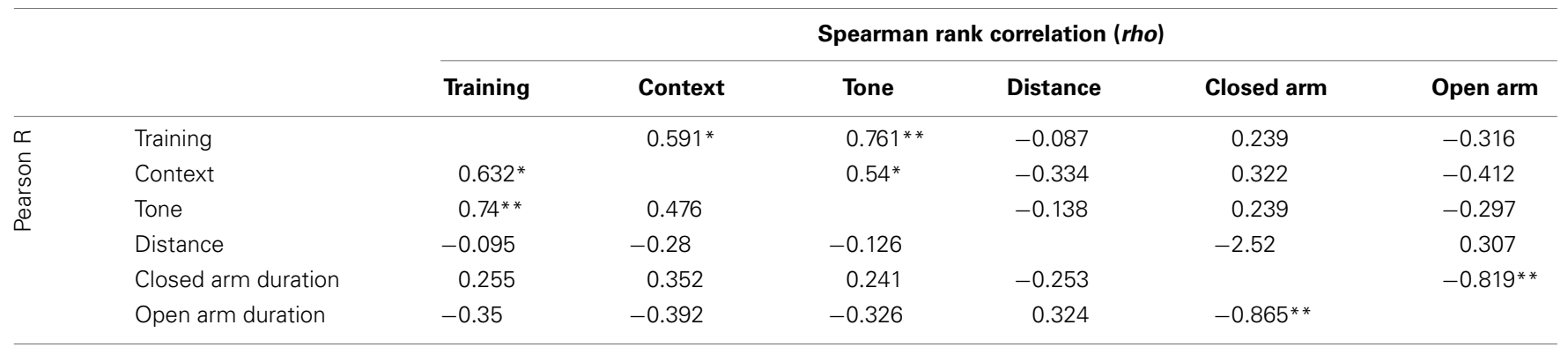

${ }^{*}$ Correlation values $>0.5,{ }^{* *}$ correlation values $>0.7$.

Table 2 | Correlation of traits measured in stress population.

Spearman rank correlation (rho)

\begin{tabular}{|c|c|c|c|c|c|c|c|}
\hline & & Training & Context & Tone & Distance & Closed arm & Open arm \\
\hline$\simeq$ & Training & & $0.63^{*}$ & $0.617^{*}$ & -0.108 & 0.139 & -0.068 \\
\hline రิ & Context & $0.651 *$ & & $0.578 *$ & 0.004 & 0.105 & -0.053 \\
\hline$\frac{1}{0}$ & Tone & $0.624 *$ & $0.562 *$ & & -0.114 & 0.175 & 0.101 \\
\hline & Distance & -0.164 & 0.077 & -0.109 & & -0.033 & 0.025 \\
\hline & Closed arm duration & 0.165 & 0.13 & 0.179 & -0.022 & & $-0.856^{* *}$ \\
\hline & Open arm duration & -0.089 & -0.052 & -0.097 & 0.052 & $-0.892 * *$ & \\
\hline
\end{tabular}

${ }^{*}$ Correlation values $>0.5,{ }^{* *}$ correlation values $>0.7$. 
strains to link regions of the genome to differences in phenotype. Potential QTLs are evaluated at regular intervals along the genome, each evaluated for significance via 2000 permutation tests (Churchill and Doerge, 1994). Thresholds for suggestive and significant QTLs are determined via GeneNetwork as likelihood ratio statistic (LRS) values associated with genome-wide probabilities of 0.67 and 0.05, respectively (Williams et al., 2001). QTLs are described here by their greatest LRS value, significance threshold passed, confidence interval (evaluated using the 1 LOD drop method; Lander and Botstein, 1989), and by the number of genes residing within the described peak. Mapping was performed for three traits of emotional learning assessed during the fear conditioning paradigm (freezing during training to tone and shock on Day 1, freezing to environmental context on Day 2, and freezing to tone on Day 3) and three emotional behavioral traits measured within the elevated plus maze [locomotion or distance $(\mathrm{m})$ traversed, time (s) in closed arms, and time (s) in open arms].

\section{CANDIDATE GENES}

Candidate genes listed are genes within each QTL which have a human homolog and/or are cis-regulating. Determination of cis-regulation was performed by using the QTLminer tool of GeneNetwork to identify cis-regulated genes (amygdala, cerebellum, hippocampus, hypothalamus, neocortex, prefrontal cortex, or striatum) within the confidence interval of each QTL, Genes were further assessed via literary search in PubMed for known relationship with stress, learning, anxiety, fear conditioning, and exploration.

\section{RESULTS}

\section{EMOTIONAL BEHAVIOR IN BXD MICE}

We assessed conditioned and unconditioned emotional behavior across BXD and parental strains using the fear conditioning paradigm and elevated plus maze, respectively. Fear learning (Figure 2) and emotional behavior (Figure 3) responses varied greatly across strains. Indeed, even the trait that showed the least variation (locomotion) from lowest to highest measured response

Table 3 | Broad-sense $\left(H^{2}\right) /$ Narrow-sense $\left(h^{2}\right)$ heritability of behavioral traits within control and chronic stress (stress) populations and the difference in behavior between control and stress (stress minus control).

\begin{tabular}{lccc}
\hline Trait & $\begin{array}{c}\text { Control } \\
\boldsymbol{H}^{\mathbf{2}} \boldsymbol{h}^{\mathbf{2}}\end{array}$ & $\begin{array}{c}\text { Stress } \\
\boldsymbol{H}^{\mathbf{2}} \boldsymbol{\boldsymbol { h } ^ { \mathbf { 2 } }}\end{array}$ & $\begin{array}{c}\text { Stress effect } \\
\boldsymbol{H}^{\mathbf{2}} \boldsymbol{\boldsymbol { h } ^ { \mathbf { 2 } }}\end{array}$ \\
\hline FEAR CONDITIONING & & & \\
Training & $0.58 / 0.35$ & $0.61 / 0.37$ & $0.27 / 0.12$ \\
Context & $0.45 / 0.25$ & $0.39 / 0.20$ & $0.20 / 0.091$ \\
Tone & $0.48 / 0.26$ & $0.46 / 0.25$ & $0.21 / 0.096$ \\
ELEVATED PLUS MAZE & & & \\
Locomotion & $0.54 / 0.32$ & $0.59 / 0.37$ & $0.24 / 0.11$ \\
Time in closed arms & $0.40 / 0.20$ & $0.32 / 0.16$ & $0.21 / 0.092$ \\
Time in open arms & $0.39 / 0.19$ & $0.29 / 0.14$ & $0.22 / 0.099$ \\
\hline
\end{tabular}

Broad-sense, VaNt; Narrow-sense, $1 / 2 \mathrm{Va} /(1 / 2 \mathrm{Va}+\mathrm{Vw}) ; \mathrm{Va}$, variance among strains; $V t$, variance across total population; $V w$, variance within strains. $(\sim 10-27)$ had a 2.5 -fold difference. The susceptibility of these traits to stress-induced effects (stress-effect) was assessed by taking the difference in the measured behavior between littermates (CVS minus control). These differences ranged from negative to positive and indicate a wide variation in stress-induced effects (Figures 2, 3, right).

All emotional behavior traits were tested for correlation in both the control (Table 1) and Stress (Table 2) population. As expected, time spent in open and closed arms during the EPM was strongly negatively correlated. Traits measuring during fear conditioning shared $R>0.48$ and rho $>0.54$ in the control condition and $R>0.56$ and rho $>0.58$ in the stress population.

\section{HERITABILITY OF EMOTIONAL BEHAVIOR}

We determined broad-sense and narrow-sense heritability to determine the proportion of variance across all strains for a measured trait that is attributable to genetic variance (Table 3 ). A $h^{2} \geq 0.25$ indicates a strong genetic component to the trait and QTL analysis was performed on those traits with a $h^{2}$ of 0.25 or greater in at least one of the populations. All measures of the fear conditioning paradigm had at least one population displaying strong heritability $\left(h^{2}=0.25-0.35\right)$. Locomotion in the elevated plus maze showed high heritability $\left(h^{2}=0.32-0.37\right)$, however time spent in closed and open arms was not determined to be strongly heritable $\left(h^{2}=0.14-0.20\right)$. Of interest, $h^{2}$ of the majority of traits decreased in CVS compared to control populations.

\section{MAIN EFFECTS ON EMOTIONAL BEHAVIOR}

To assess the effect of stress on emotional behaviors measured across the BXD strains, we first ran a non-linear mixed-effect test followed by ANOVA for effects of stress and strain (Table 4). An effect for strain was seen in all measures, indicating that the unique genetic background of the different strains determined the measured behavioral response, consistent with our measures of heritability. Significant effects of stress were seen in freezing

Table 4 | Summary of One-Way ANOVA results following linear mixed model fit.

\begin{tabular}{|c|c|c|c|}
\hline Trait & & $\begin{array}{l}\text { Strain } \\
P, F, d f\end{array}$ & $\begin{array}{l}\text { Stress } \\
P, F, d f\end{array}$ \\
\hline \multicolumn{4}{|c|}{ FEAR CONDITIONING } \\
\hline & Training & $\begin{array}{r}{ }^{*}<0.001 \\
5.41,63\end{array}$ & $\begin{array}{c}*<0.0001, \\
16.05,1\end{array}$ \\
\hline & Context & $\begin{array}{c}{ }^{*}<0.0001 \\
2.80,63\end{array}$ & $\begin{array}{r}*<0.05 \\
4.96,1\end{array}$ \\
\hline & Tone & $\begin{array}{c}{ }^{*}<0.0001 \\
6.50,63\end{array}$ & $\begin{array}{r}* 0.0001, \\
26.22,1\end{array}$ \\
\hline \multicolumn{4}{|c|}{ ELEVATED PLUS MAZE } \\
\hline & Locomotion & $\begin{array}{c}{ }^{*}<0.0001 \\
10.15,63\end{array}$ & $\begin{array}{l}0.35 \\
0.92,1\end{array}$ \\
\hline & $\begin{array}{l}\text { Time in } \\
\text { closed arms }\end{array}$ & $\begin{array}{c}{ }^{*}<0.0001 \\
2.04,63\end{array}$ & $\begin{array}{r}*<0.05 \\
4.23,1\end{array}$ \\
\hline & $\begin{array}{l}\text { Time in } \\
\text { open arms }\end{array}$ & $\begin{array}{c}{ }^{*}<0.001 \\
1.92,63\end{array}$ & $\begin{array}{l}0.65 \\
0.22,1\end{array}$ \\
\hline
\end{tabular}

*indicates significant $p$-value. 
to training (Day 1), context (Day 2), and tone (Day 3) in the fear conditioning paradigm and time in open and closed arms of the elevated plus maze, indicating that these behavioral responses were susceptible to chronic stress exposure. Additional environmental factors were tested as confounds in mixed model analysis (month of testing and handler during testing; Valdar et al., 2006). Month of testing did not hold a main effect for any trait tests, while handler was a significant factor in time spent in closed arms during the EPM alone $P<0.01(F=6.86, d f=3)$.

\section{OTL MAPPING LINKS EMOTIONAL PHENOTYPES TO CAUSAL LOCI}

QTLs were detected in freezing to training (Day 1), context (Day 2), and tone (Day 3), and locomotion (Tables 5, 6). No QTLs meeting a suggestive level of LRS significance were found for time spent in open and closed arms of the EPM. The lack of QTLs for time spent in EPM is consistent with the non-significant $h^{2}$ scores reported for these measures. Additionally, QTLs for the Stress-Effect on locomotion and freezing to training (Day 1) were detected. QTL maps of all measured traits having suggestive or significant LRS peaks are summarized in Table 3 and displayed as a heatmap (Figure 4). The use of a heatmap allows for comparison across phenotypic traits and the presence of stress. Individual QTL maps containing suggestive and significant peaks for the control and CVS populations are found in Figures 5, 6, respectively.

For reference, QTLs are denoted by chromosome location (Table 5). QTLs unique to the control population were located on Chromosomes 13 and 14 (QTLs 13a and 14) and were found in the analysis of freezing to context (Day 2) and tone (Day 3), respectively. Conversely, QTL mapping of freezing to training (Day 1) uncovered QTLs unique to the CVS population on Chromosomes 2 and 5. All other QTLs (QTLs 1, 3, 4, 13b, and $\mathrm{X}$ ) were shared by both control and CVS populations. QTLs 1, 3 , and $13 \mathrm{~b}$ were identified in both control and CVS populations for freezing to training. QTL 13b was also identified in freezing to context (Day 2) for both control and CVS populations and freezing to tone (Day 3) in the CVS population. Lastly, QTL 4 was identified in both control and CVS populations for locomotion in the elevated plus maze. Candidate genes within each QTL are listed in Table 6.

\section{DISCUSSION}

We observed significant effects of chronic stress on emotional behavioral phenotypes. The BXD populations utilized revealed great variability of behavioral response in both control and CVS conditions (Figures 1, 2). Thus, our results show that environmental stress is a unique factor affecting behavioral responses in BXD strains of mice.

QTL mapping of individual phenotypes identified genetic loci that predict emotional behavior in control and stress environments (Table 5). The unique QTL maps from control vs. chronic stress populations for the same behavioral attribute suggest that the genetic regions most likely to predict behavior are influenced by prior stress history. Of interest, the strength of each trait's heritability score lessened with the presence of chronic stress, suggesting a disruption of the

\section{Table 5 | List of QTLs.}

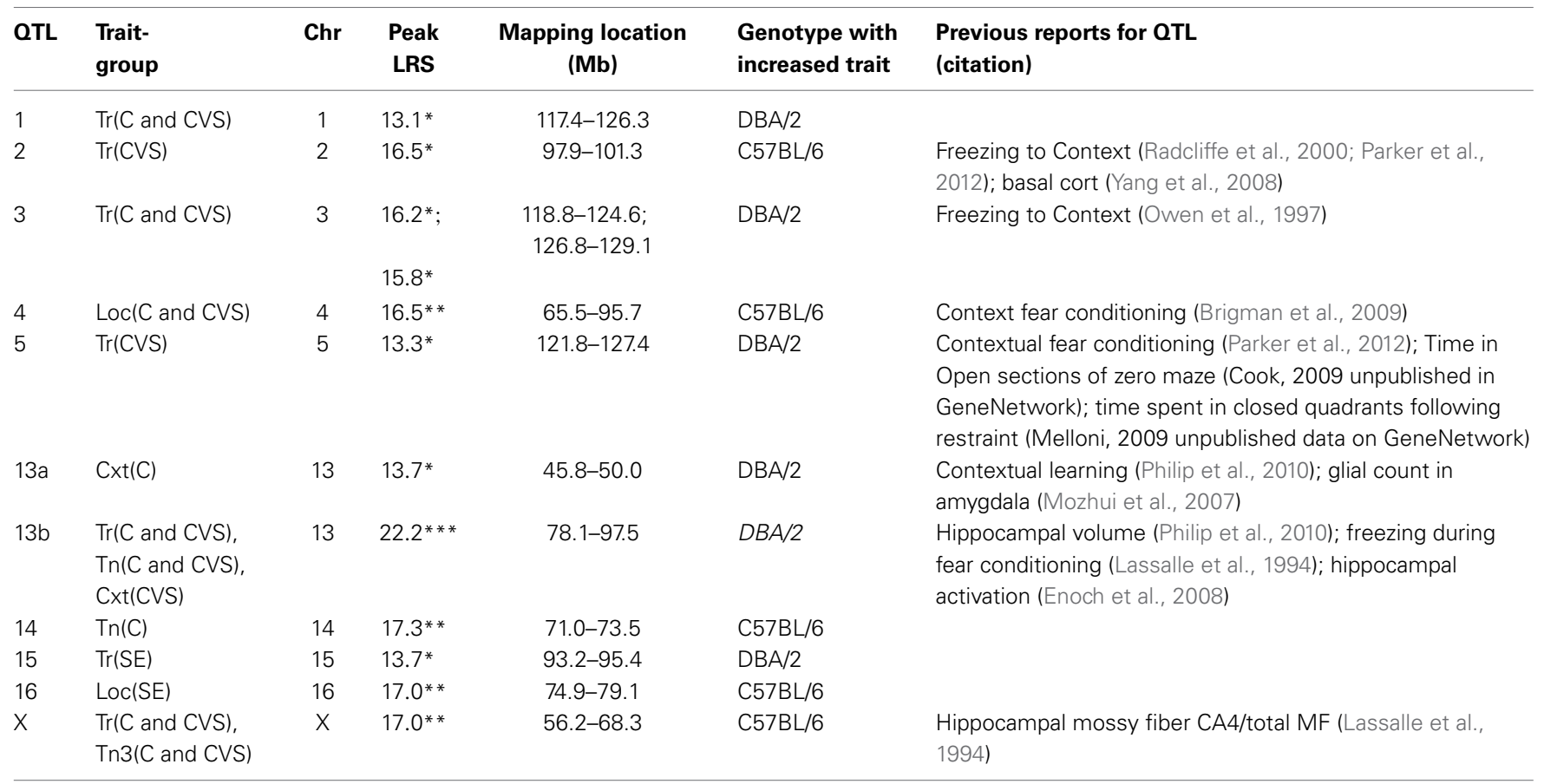

Trait/group abbreviations: C, control; CVS, chronic variable stress; Chr, chromosome; SE, stress-effect; Tr, freezing to training; Cxt, freezing to context; Th, freezing to tone; and Loc, locomotion.

LRS: greatest LRS reported. Significant level: * suggestive, ${ }^{* *}$ significant, ${ }^{* * *}$ highly significant per genenetwork.org. 
Table 6 | List of candidate genes.

\begin{tabular}{|c|c|c|c|}
\hline QTL & Chr & \# Genes (candidate/total) & Candidate genes \\
\hline 2 & 2 & $0 / 1$ & \\
\hline 3 & 3 & $30 / 55$ & 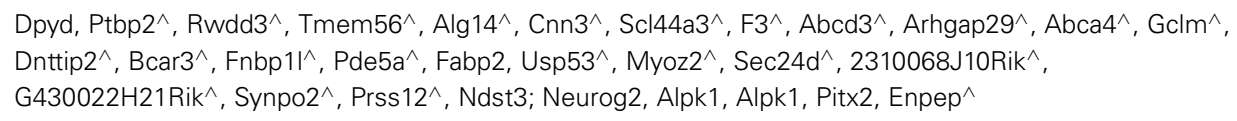 \\
\hline 4 & 4 & $60 / 115$ & 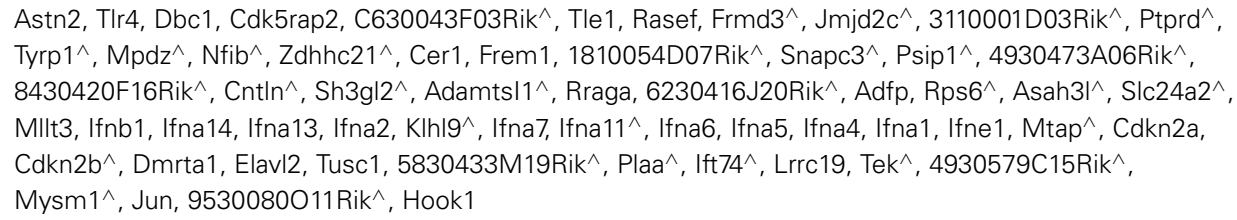 \\
\hline
\end{tabular}

55 57/112 Trafd1, Mapkap5, Aldh2, Acad10, Brap^^,Atxn2, Myl2, Ppp1cc, Rad9b, Vps29^ Arpc3, Anapc7, Atp2a2,
P2rx7, P2rx4, Camkk2, Anapc5, Rnf34, Fbxl10, Hpd, Psmd9^, Wdr66^, Bcl7a, Mixip^, II31, Lrrc43, B3gnt4, Diablo^, Vps33a, Clip1^, Zcchc8, Kntc1^, Gpr109a, Gpr81, Denr, Hip1r, Abcb9, Arl6ip4, Pitpnm2, Mphosph9^, Cdk2ap1^, Sbno1^, Ddx55^, Eif2b1, Gtf2h3, Atp6v0a2, Ccdc92^, Zfp664^, 3110032G18Rik^,

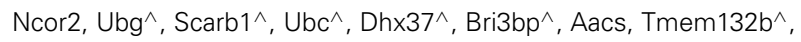

\begin{tabular}{|c|c|c|c|}
\hline $13 a$ & 13 & $27 / 57$ & 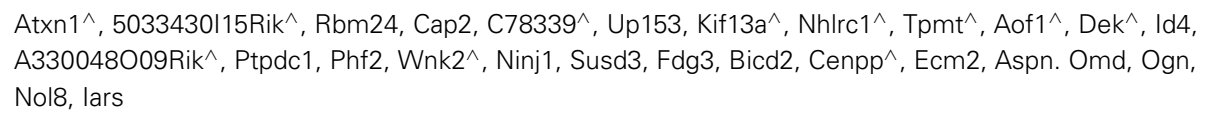 \\
\hline 14 & 14 & $6 / 15$ & Fgf17, Npm2^ ${ }^{\wedge}$, Xpo $^{\wedge}{ }^{\prime}$, Dok2^${ }^{\wedge}$, Gfra2^${ }^{\wedge}$, Cysltr2 \\
\hline 15 & 15 & $9 / 12$ & 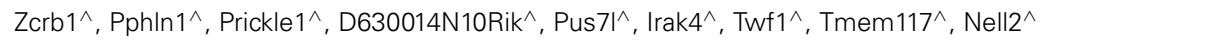 \\
\hline 16 & 16 & $14 / 29$ & 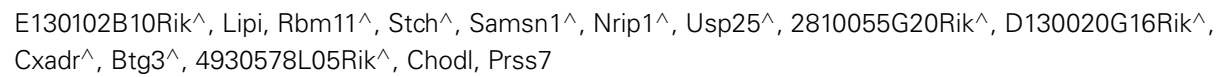 \\
\hline
\end{tabular}

Candidate genes were defined as genes with a human homolog and/or are cis-regulated: $\wedge^{\wedge}$ indicates cis-regulated gene.

basal heritable contribution with chronic stress. Our mixedmodel analysis identified behavioral traits susceptible to stressinduced effects that were seen in differences in their resulting QTL maps (Table 4). Most QTLs for the behavioral phenotypes reported here have not been previously indentified for these traits, although several have been identified for related phenotypes (Table 5). As the initial report of these QTLs and candidate genes, additional studies are required to strengthen their validation.

\section{OTLS AND CANDIDATE GENES FOR LEARNING WITHIN A STRESSFUL CONTEXT}

QTL 13b was found repeatedly for fear learning in both control and chronic stress populations. Although its strength and frequency is greater with chronic stress, the region was not identified for anxiety/exploratory EPM behavior. Its presence in freezing to training (Day 1) and tone (Day 3) of the control population suggests pleiotropic effects for learning within a stressful context. QTL 13b corresponds with peaks found previously for fear conditioning and hippocampal volume (Table 5; Lassalle et al., 1994; Philip et al., 2010; Parker et al., 2012). The myocyte-specific enhancer binding factor $2 \mathrm{C}(\mathrm{Mef} 2 \mathrm{c})$ gene resides at the highest LRS value within QTL 13b, and is cis-regulated. Gene expression of $M e f 2 c$ in the amygdala has been associated with anxiety (Genenetowrk GeneWiki: Williams et al., 2001), and plays a role in synapse elimination within the hippocampus to facilitate hippocampal dependent learning (Barbosa et al., 2008). Additionally, Mef2c has been identified as a requirement for the activationdependent expression of BDNF (Lyons et al., 2012) and is a marker of ischemia-resistant hippocampal neurons (Speliotes et al., 1996). Mutations of Mef2c in humans result in mental retardation (Zweier et al., 2010). Taken together, its presence in QTL 


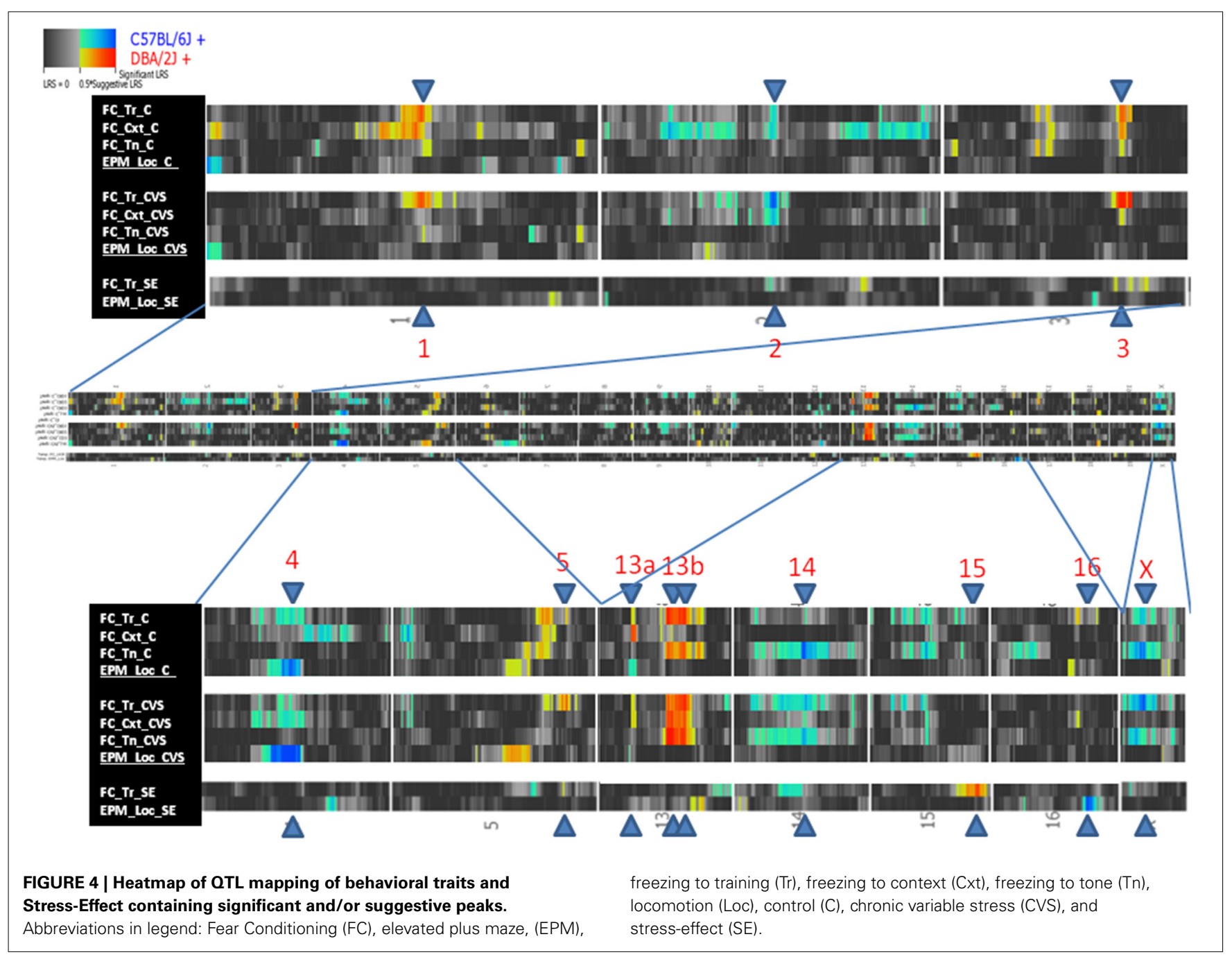

$13 \mathrm{~b}$ and its known associations suggest that it may have a role during stress and/or anxiety.

Relevant to learning and stress, corticotropin-releasing hormone binding protein (Crhbp) is also found within QTL $13 \mathrm{~b}$. Crhbp regulates the activity of $\mathrm{CRH}$ (corticotropin releasing hormone), a stress hormone in the HPA axis (Westphal and Seasholtz, 2006), and is upregulated following stress (McClennen et al., 1998). The HPA axis has a complex relationship with learning and memory- transient activation results in enhancement of learning and memory (de Kloet et al., 1999), while persistent activation levels results in cognitive deficit (de Kloet et al., 2005). Enoch et al. reported that dense whole genome linkage scan of hippocampal activation assessed by EEG resulted in a linkage peak containing crhbp (Enoch et al., 2008). Crhbp is found at greater levels in high avoidance rats compared to low avoidance rats (Sabariego et al., 2011). Additionally, mutations in Crhbp were associated with anxiety disorders in a Plains Indian population and alcohol use in Caucasians, suggesting a role for Crhbp in stress-induced phenotypes (Enoch et al., 2008). These results suggest Crhbp may be a marker for fear learning in a stress context.
Also associated with fear learning in both control and chronic stress populations, QTL X was identified for the freezing to training and tone (Days 1 and 3) and absent for context (Day 2 ). Genes within this region may play a role in the incorporation of the auditory cue and aversive stimulus. Fibroblast growth factor 13 (Fgf13) is located at the LRS peak of QTL X and has a well described role in learning and memory (Wu et al., 2012). Expressed in cortical neurons, Fgf13 is linked to X-chromosomelinked mental retardation in humans (Itoh and Ornitz, 2008). Interestingly, the expression of the Mef2c (QTL 13b) and Fgf13 in the hippocampus are positively correlated (genenetwork.org, Rho $=0.543, P=0.00356$ ). Thus, genes in both QTLs 13b and X may interact for the expression of learning phenotypes.

QTL 5 was unique to the stress population for freezing to training (Day 1) and has been found previously (Table 5; Parker et al., 2012; Cook, 2009 unpublished data in genenetwork.org) Within QTL 5, P2rx7, and P2rx4 have roles in neuroinflammatory response to stress (Witting et al., 2004; Hernandez et al., 2010). Knockout and antagonism of $P 2 r x 7$ results in mood stabilizing and reduces the corticosterone response to restraint stress (Csolle et al., 2013). Calcium/calmoudulin kinase kinase 2 (Camkk2), a 


\section{A Freezing to Training}

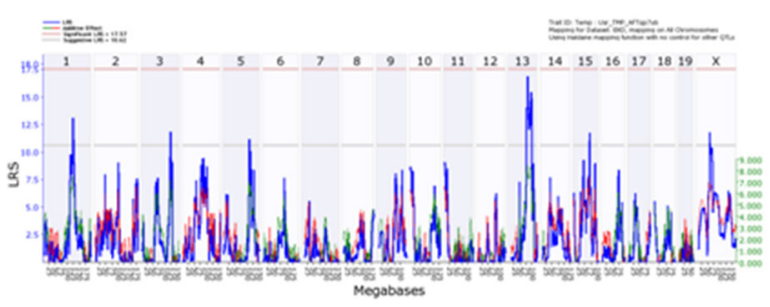

\section{c Freezing to Tone}

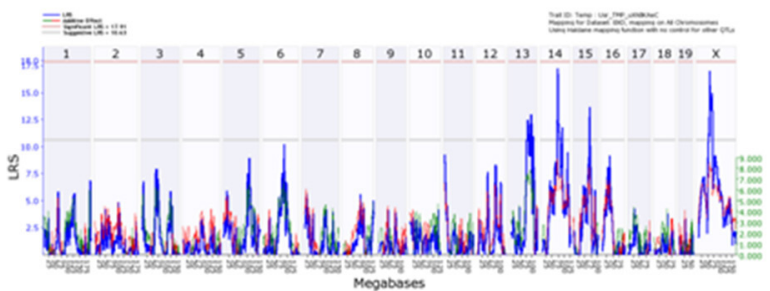

\section{B Freezing to Context}

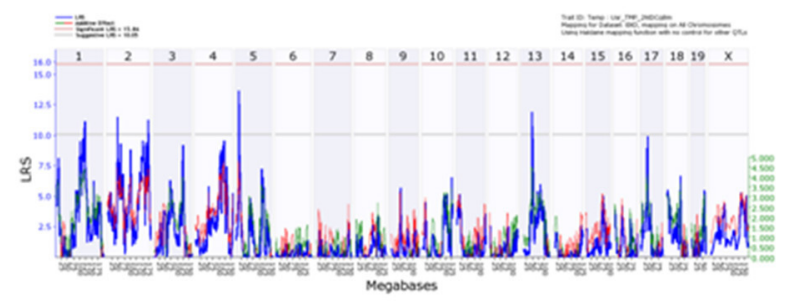

\section{Locomotion}

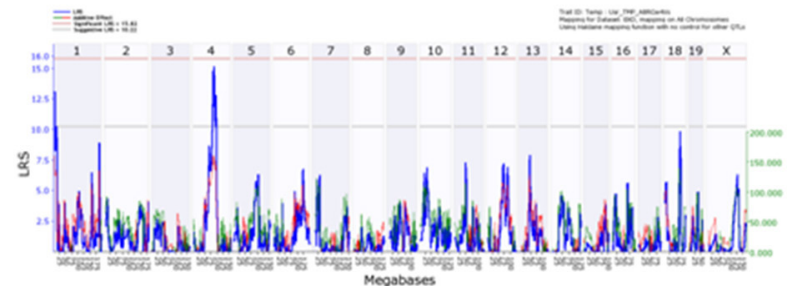

FIGURE 5 | Individual QTL maps of control population phenotypic traits. (A) Freezing to Training (Day 1), (B) Freezing to Context (Day 2), (C) Freezing to Tone (Day 3), (D) Locomotion.

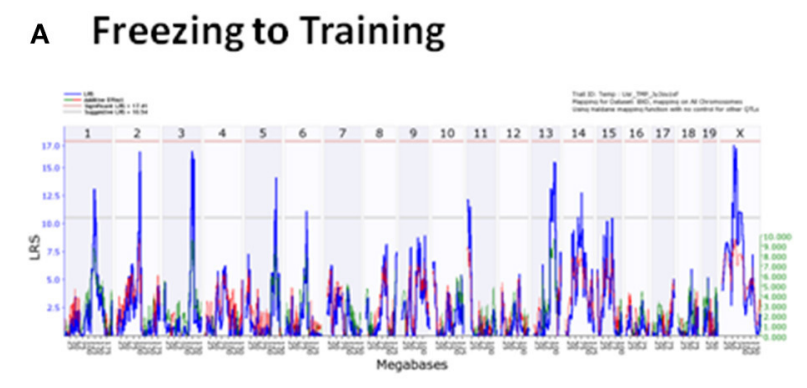

c Freezing to Tone

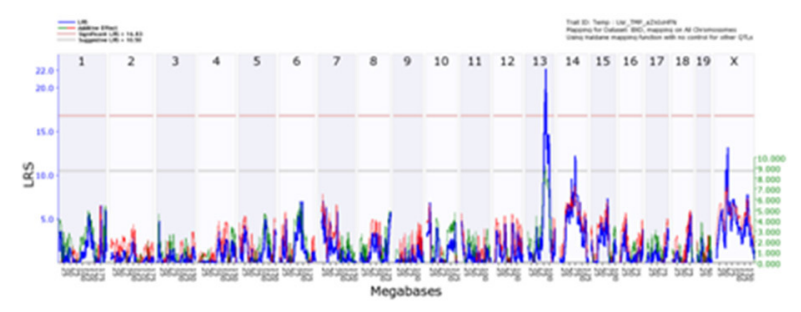

\section{B Freezing to Context}

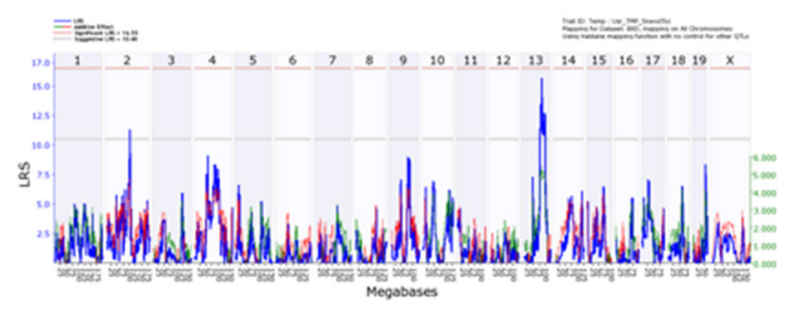

D Locomotion

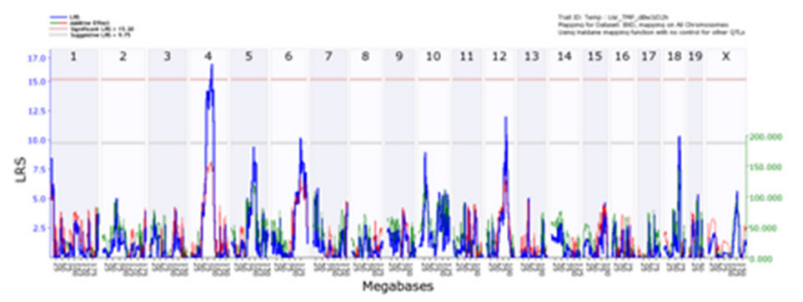

FIGURE 6 | Individual QTL maps of chronic stress population phenotypic traits. (A) Freezing to Training (Day 1), (B) Freezing to Context (Day 2), (C) Freezing to Tone (Day 3), (D) Locomotion.

gene associated with hippocampus-dependent long-term memory and anxiety also resides in QTL 5 (Peters et al., 2003; Mizuno et al., 2007; Sabariego et al., 2011). Loss of Camkk2 decreases BDNF expression (Kokubo et al., 2009). Interestingly, fear conditioning results in a downregulation of Camkk2 in the hippocampus (Mei et al., 2005) and Camkk2 knock outs males, but not females, show impaired spatial learning and normal fear conditioning (Peters et al., 2003; Mizuno et al., 2007). Our experiments, using only male BXD mice, confirm a contributory role of Camkk2 in fear learning.

QTL analysis of the Stress-Effect revealed a significant peak for difference in locomotion (QTL 16) and suggestive peak for difference in freezing to training (Day 1) (QTL 15). These genetic regions contain Nrip1 and Nell2, two genes required for learning of the Morris Water Maze (Duclot et al., 2012) (Matsuyama et al., 2005). Of note, swimming aberrations were noted as a potential 
contributor to the cognitive deficit, despite the absence of difference in rotorod and open field performance (Duclot et al., 2012). Elimination of Nell2 via knockout results in increases in LTP within the dentate gyrus (Matsuyama et al., 2004), suggesting that its role in cognitive performance is due to a regulatory effect on LTP.

\section{OTLS AND CANDIDATE GENES FOR LEARNING IN A CONTROL ENVIRONMENT}

Unique to learning in the control environment, QTLs 13a and 14 expectedly contained genes associated with learning. No genes with established rolesin stress or anxiety were found. QTL 13a, previously found for contextual learning and amgdala glial count (Table 5; Mozhui et al., 2007; Philip et al., 2010) contains NHL repeat containing 1/epilepsy, progressive myoclonic 2B (Nhlrc1/EPM2B). Knockout of this gene results in episodic memory deficits assessed by the object recognition test (GarciaCabrero et al., 2012). Thus, Nhlrc1 may play a role in the incorporation or retrieval of contextual memory. Learning an auditory cue (freezing to tone- Day 3) resulted in a significant QTL on Chromosome 14 in controls. Several genes within this locus are associated with hippocampal-dependent learning [e.g., Gfra, Itm2B, and Htr2a (Voikar et al., 2004; Tamayev et al., 2010; Zhang et al., 2013)]. Loss of the QTLs with chronic stress may indicate that genes within QTLs 13a and 14 no longer play a significant role when stress is present, or that genes within other genetic loci play overshadowing roles with stress. Of note, the expression of Nhlrc1 in QTL 13a, and Itm $2 b$ and Htr2a in QTL 14 in the whole brain correlate positively with whole brain expression of the gene Slc24a2 in QTL 4 (genenetwork.org, Rho $=0.612, p<0.00037$, Rho $=0.549, p<0.0020$, and Rho $=0.581, p<0.00089$, respectively). This is of interest because these four genes have been associated with learning and may act in concert to affect learning in their respective phenotypes.

The results from this study identified genetic loci for emotional behavioral phenotypes in the presence and absence of chronic stress. Four of the loci identified in this study are novel and their presence across multiple traits supports further study of the candidate genes contained within. In sum, our data indicate that distinct genetic loci are associated with emotional behavior in control and chronic stress conditions suggesting that behavioral outcomes are influenced by the interplay between prior stress history and genetic background.

\section{ACKNOWLEDGMENTS}

We would like to express our appreciation to TSgt Laura Bass, Naomi Bechmann, Dr. Megan Durbin, Dr. Catherine Harrison, Benjamin Holmes, Craig Hughes, TSgt Andrew Jimenez, Dr. Erica Johnson, Dr. Tom Lamkin, 1st Lt Danielle McCarty, Raquel Moore, 1st Lt Wilson Tucker, and Jessica Wagner without whom this work could not have been completed. In addition, we would like to thank Dr. Stephen C. Benoit for review of manuscript and meaningful discussion. This work was supported by Air Force Office of Scientific Research/Air Force Research Laboratory (AFOSR/AFRL). Distribution A: approved for public release; distribution unlimited. 88ABW Cleared 02/26/2014; 88ABW-2014-0757.

\section{REFERENCES}

Andreux, P. A., Williams, E. G., Koutnikova, H., Houtkooper, R. H., Champy, M. F., Henry, H., et al. (2012). Systems genetics of metabolism: the use of the BXD murine reference panel for multiscalar integration of traits. Cell 150, 1287-1299. doi: 10.1016/j.cell.2012.08.012

Barbosa, A. C., Kim, M. S., Ertunc, M., Adachi, M., Nelson, E. D., McAnally, J., et al. (2008). MEF2C, a transcription factor that facilitates learning and memory by negative regulation of synapse numbers and function. Proc. Natl. Acad. Sci. U.S.A. 105, 9391-9396. doi: 10.1073/pnas.0802679105

Bienvenu, O. J., Davydow, D. S., and Kendler, K. S. (2011). Psychiatric 'diseases' versus behavioral disorders and degree of genetic influence. Psychol. Med. 41, 33-40. doi: 10.1017/S003329171000084X

Binder, E. B. (2012). The genetic basis of mood and anxiety disorders changing paradigms. Biol. Mood Anxiety Disord. 2:17. doi: 10.1186/2045-53 80-2-17

Brigman, J. L., Mathur, P., Lu, L., Williams, R. W., and Holmes, A. (2009). Genetic relationship between anxiety-related and fear-related behaviors in BXD recombinant inbred mice. Behav. Pharmacol. 20, 204-209. doi: 10.1097/FBP.0b013e32830c368c

Castañeda, T. R., Noqueiras, R., Müller, T. D., Krishna, R., Grant, E., Jones, A., et al. (2011). Decreased glucose tolerance and plasma adiponectin: resistin ration in a mouse model of post-traumatic stress disorder. Diabetologia 54, 900-909. doi: 10.1007/s00125-010-2019-y

Chernomas, W. M., and Shapiro, C. (2013). Stress, depression, and anxiety among undergraduate nursing students. Int. J. Nurs. Educ. Scholarsh. 10, 255-266. doi: 10.1515/ijnes-2012-0032

Churchill, G. A., and Doerge, R. W. (1994). Empirical threshold values for quantitative trait mapping. Genetics 138, 963-971.

Csolle, C., Ando, R. D., Kittel, A., Goloncser, F., Baranyi, M., Soproni, K., et al. (2013). The absence of P2X7 receptors (P2rx7) on non-haematopoietic cells leads to selective alteration in mood-related behaviour with dysregulated gene expression and stress reactivity in mice. Int. J. Neuropsychopharmacol. 16, 213-233. doi: 10.1017/S1461145711001933

de Kloet, E. R., Joels, M., and Holsboer, F. (2005). Stress and the brain: from adaptation to disease. Nat. Rev. Neurosci. 6, 463-475. doi: 10.1038/ nrn1683

de Kloet, E. R., Oitzl, M. S., and Joels, M. (1999). Stress and cognition: are corticosteroids good or bad guys? Trends Neurosci. 22, 422-426. doi: 10.1016/S01662236(99)01438-1

Duclot, F., Lapierre, M., Fritsch, S., White, R., Parker, M. G., Maurice, T., et al. (2012). Cognitive impairments in adult mice with constitutive inactivation of RIP140 gene expression. Genes Brain Behav. 11, 69-78. doi: 10.1111/j.1601183X.2011.00731.x

Enoch, M. A., Shen, P. H., Ducci, F., Yuan, Q., Liu, J., White, K. V., et al. (2008). Common genetic origins for EEG, alcoholism and anxiety: the role of CRH-BP. PLoS ONE 3:e3620. doi: 10.1371/journal.pone.0003620

Furay, A. R., Bruestle, A. E., and Herman, J. P. (2008). The role of the forebrain glucocorticoid receptor in acute and chronic stress. Endocrinology 149, 5482-5490. doi: 10.1210/en.2008-0642

Garcia-Cabrero, A. M., Marinas, A., Guerrero, R., de Cordoba, S. R., Serratosa, J. M., and Sanchez, M. P. (2012). Laforin and malin deletions in mice produce similar neurologic impairments. J. Neuropathol. Exp. Neurol. 71, 413-421. doi: 10.1097/NEN.0b013e318253350f

Herman, J. P., and Watson, S. J. (1995). Stress regulation of mineralocorticoid receptor heteronuclear RNA in rat hippocampus. Brain Res. 677, 243-249. doi: 10.1016/0006-8993(95)00152-G

Hernandez, S., Casanovas, A., Piedrafita, L., Tarabal, O., and Esquerda, J. E. (2010). Neurotoxic species of misfolded SOD1G93A recognized by antibodies against the P2X4 subunit of the ATP receptor accumulate in damaged neurons of transgenic animal models of amyotrophic lateral sclerosis. J. Neuropathol. Exp. Neurol. 69, 176-187. doi: 10.1097/NEN.0b013e3181cd3e33

Itoh, N., and Ornitz, D. M. (2008). Functional evolutionary history of the mouse Fgf gene family. Dev. Dyn. 237, 18-27. doi: 10.1002/dvdy.21388

Kokubo, M., Nishio, M., Ribar, T. J., Anderson, K. A., West, A. E., and Means, A. R. (2009). BDNF-mediated cerebellar granule cell development is impaired in mice null for CaMKK2 or CaMKIV. J. Neurosci. 29, 8901-8913. doi: 10.1523/JNEUROSCI.0040-09.2009

Lander, E. S., and Botstein, D. (1989). Mapping mendelian factors underlying quantitative traits using RFLP linkage maps. Genetics 121, 185-199. 
Lassalle, J. M., Halley, H., and Roullet, P. (1994). Analysis of behavioral and hippocampal variation in congenic albino and pigmented BALB mice. Behav. Genet. 24, 161-169. doi: 10.1007/BF01067820

Lyons, M. R., Schwarz, C. M., and West, A. E. (2012). Members of the myocyte enhancer factor 2 transcription factor family differentially regulate Bdnf transcription in response to neuronal depolarization. J. Neurosci. 32, 12780-12785. doi: 10.1523/JNEUROSCI.0534-12.2012

Matsuyama, S., Aihara, K., Nishino, N., Takeda, S., Tanizawa, K., Kuroda, S., et al. (2004). Enhanced long-term potentiation in vivo in dentate gyrus of NELL2deficient mice. Neuroreport 15, 417-420. doi: 10.1097/00001756-20040301000007

Matsuyama, S., Doe, N., Kurihara, N., Tanizawa, K., Kuroda, S., Iso, H., et al. (2005). Spatial learning of mice lacking a neuron-specific epidermal growth factor family protein, NELL2. J. Pharmacol. Sci. 98, 239-243. doi: 10.1254/jphs.FP0050211

McClennen, S. J., Cortright, D. N., and Seasholtz, A. F. (1998). Regulation of pituitary corticotropin-releasing hormone-binding protein messenger ribonucleic acid levels by restraint stress and adrenalectomy. Endocrinology 139, 4435-4441.

Mei, B., Li, C., Dong, S., Jiang, C. H., Wang, H., and Hu, Y. (2005). Distinct gene expression profiles in hippocampus and amygdala after fear conditioning. Brain Res. Bull. 67, 1-12. doi: 10.1016/j.brainresbull.2005.03.023

Mizuno, K., Antunes-Martins, A., Ris, L., Peters, M., Godaux, E., and Giese, K. P. (2007). Calcium/calmodulin kinase kinase beta has a malespecific role in memory formation. Neuroscience 145, 393-402. doi: 10.1016/j.neuroscience.2006.11.056

Mozhui, K., Hamre, K. M., Holmes, A., Lu, L., and Williams, R. W. (2007). Genetic and structural analysis of the basolateral amygdala complex in BXD recombinant inbred mice. Behav. Genet. 37, 223-243. doi: 10.1007/s10519-0069122-3

Mozhui, K., Karlsson, R. M., Kash, T. L., Ihne, J., Norcross, M., Patel, S., et al. (2010). Strain differences in stress responsivity are associated with divergent amygdala gene expression and glutamate-mediated neuronal excitability. J. Neurosci. 30, 5357-5367. doi: 10.1523/JNEUROSCI.5017-09.2010

Owen, E. H., Christensen, S. C., Paylor, R., and Wehner, J. M. (1997). Identification of quantitative trait loci involved in contextual and auditory-cued fear conditioning in BXD recombinant inbred strains. Behav. Neurosci. 111, 292-300. doi: 10.1037/0735-7044.111.2.292

Pardon, M. C., Perez-Diaz, F., Joubert, C., and Cohen-Salmon, C. (2000a). Influence of a chronic ultramild stress procedure on decision-making in mice. J. Psychiatry Neurosci. 25, 167-177.

Pardon, M., Perez-Diaz, F., Joubert, C., and Cohen-Salmon, C. (2000b). Agedependent effects of a chronic ultramild stress procedure on open-field behaviour in B6D2F1 female mice. Physiol. Behav. 70, 7-13. doi: 10.1016/S00319384(00)00216-X

Parker, C. C., Sokoloff, G., Cheng, R., and Palmer, A. A. (2012). Genome-wide association for fear conditioning in an advanced intercross mouse line. Behav. Genet. 42, 437-448. doi: 10.1007/s10519-011-9524-8

Peirce, J. L., Lu, L., Gu, J., Silver, L. M., and Williams, R. W. (2004). A new set of $\mathrm{BXD}$ recombinant inbred lines from advanced intercross populations in mice. BMC Genet. 5:7. doi: 10.1186/1471-2156-5-7

Peters, M., Mizuno, K., Ris, L., Angelo, M., Godaux, E., and Giese, K. P. (2003). Loss of $\mathrm{Ca} 2+/$ calmodulin kinase kinase beta affects the formation of some, but not all, types of hippocampus-dependent long-term memory. J. Neurosci. 23, 9752-9760.

Philip, V. M., Duvvuru, S., Gomero, B., Ansah, T. A., Blaha, C. D., Cook, M. N., et al. (2010). High-throughput behavioral phenotyping in the expanded panel of BXD recombinant inbred strains. Genes Brain Behav. 9, 129-159. doi: 10.1111/j.1601-183X.2009.00540.x

Powell, N. D., Sloan, E. K., Bailey, M. T., Arevalo, J. M., Miller, G. E., Chen, E., et al. (2013). Social stress up-regulates inflammatory gene expression in the leukocyte transcriptome via beta-adrenergic induction of myelopoiesis. Proc. Natl. Acad. Sci. U.S.A. 110, 16574-16579. doi: 10.1073/pnas.1310655110

Radcliffe, R. A., Lowe, M. V., and Wehner, J. M. (2000). Confirmation of contextual fear conditioning QTLs by short-term selection. Behav. Genet. 30, 183-191. doi: 10.1023/A:1001910107167

Reijneveld, S. A., and Schene, A. H. (1998). Higher prevalence of mental disorders in socioeconomically deprived urban areas in The Netherlands: community or personal disadvantage? J. Epidemiol. Community Health 52, 2-7. doi: 10.1136/jech.52.1.2
Renoir, T., Hasebe, K., and Gray, L. (2013). Mind and body: how the health of the body impacts on neuropsychiatry. Front. Pharmacol. 4:158. doi: 10.3389/fphar.2013.00158

Sabariego, M., Gomez, M. J., Moron, I., Torres, C., Fernandez-Teruel, A., Tobena, A., et al. (2011). Differential gene expression between inbred Roman high(RHA-I) and low- (RLA-I) avoidance rats. Neurosci. Lett. 504, 265-270. doi: 10.1016/j.neulet.2011.09.044

Speliotes, E. K., Kowall, N. W., Shanti, B. F., Kosofsky, B., Finklestein, S. P., and Leifer, D. (1996). Myocyte-specific enhancer binding factor 2C expression in gerbil brain following global cerebral ischemia. Neuroscience 70, 67-77. doi: 10.1016/0306-4522(95)00301-X

Tamayev, R., Matsuda, S., Fa, M., Arancio, O., and D’Adamio, L. (2010). Danish dementia mice suggest that loss of function and not the amyloid cascade causes synaptic plasticity and memory deficits. Proc. Natl. Acad. Sci. U.S.A. 107, 20822-20827. doi: 10.1073/pnas.1011689107

Valdar, W., Solberg, L. C., Gauguier, D., Cookson, W. O., Rawlins, J. N., Mott, R., et al. (2006). Genetic and environmental effects on complex traits in mice. Genetics 174, 959-984. doi: 10.1534/genetics.106.060004.

Valentinuzzi, V. S., Kolker, D. E., Vitaterna, M. H., Shimomura, K., Whiteley, A., Low-Zeddies, S., et al. (1998). Automated measurement of mouse freezing behavior and its use for quantitative trait locus analysis of contextual fear conditioning in $(\mathrm{BALB} / \mathrm{cJ} \times \mathrm{C} 57 \mathrm{BL} / 6 \mathrm{~J}) \mathrm{F} 2$ mice. Learn. Mem. 5, 391-403.

Voikar, V., Rossi, J., Rauvala, H., and Airaksinen, M. S. (2004). Impaired behavioural flexibility and memory in mice lacking GDNF family receptor alpha2. Eur. J. Neurosci. 20, 308-312. doi: 10.1111/j.1460-9568.2004.03475.x

Waddell, J., Dunnett, C., and Falls, W. A. (2004). C57BL/6J and DBA/2J mice differ in extinction and renewal of extinguished conditioned fear. Behav. Brain Res. 154, 567-576. doi: 10.1016/j.bbr.2004.03.025

Westphal, N. J., and Seasholtz, A. F. (2006). CRH-BP: the regulation and function of a phylogenetically conserved binding protein. Front. Biosci. 11, 1878-1891. doi: 10.2741/1931

Williams, R., Yan, L., Sloan, Z., and Centeno, A. (2001). GeneNetwork WebQTL. Available online at: www.genenetwork.org

Witting, A., Walter, L., Wacker, J., Moller, T., and Stella, N. (2004). P2X7 receptors control 2-arachidonoylglycerol production by microglial cells. Proc. Natl. Acad. Sci. U.S.A. 101, 3214-3219. doi: 10.1073/pnas.0306707101

Wu, Q. F., Yang, L., Li, S., Wang, Q., Yuan, X. B., Gao, X., et al. (2012). Fibroblast growth factor 13 is a microtubule-stabilizing protein regulating neuronal polarization and migration. Cell 149, 1549-1564. doi: 10.1016/j.cell.2012.04.046

Yang, R. J., Mozhui, K., Karlsson, R. M., Cameron, H. A., Williams, R. W., and Holmes, A. (2008). Variation in mouse basolateral amygdala volume is associated with differences in stress reactivity and fear learning. Neuropsychopharmacology 33, 2595-2604. doi: 10.1038/sj.npp.1301665

Zhang, C., Wang, L., Liao, Q., Zhang, L., Xu, L., Chen, C., et al. (2013). Genetic associations with hypertension: meta-analyses of six candidate genetic variants. Genet. Test Mol. Biomarkers 17, 736-742. doi: 10.1089/gtmb. 2013.0080

Zweier, M., Gregor, A., Zweier, C., Engels, H., Sticht, H., Wohlleber, E., et al. (2010). Mutations in MEF2C from the 5q14.3q15 microdeletion syndrome region are a frequent cause of severe mental retardation and diminish MECP2 and CDKL5 expression. Hum. Mutat. 31, 722-733. doi: 10.1002/humu.21253

Conflict of Interest Statement: The authors declare that the research was conducted in the absence of any commercial or financial relationships that could be construed as a potential conflict of interest.

Received: 23 May 2014; accepted: 11 September 2014; published online: 21 October 2014.

Citation: Carhuatanta KAK, Shea CJA, Herman JP and Jankord R (2014) Unique genetic loci identified for emotional behavior in control and chronic stress conditions. Front. Behav. Neurosci. 8:341. doi: 10.3389/fnbeh.2014.00341

This article was submitted to the journal Frontiers in Behavioral Neuroscience.

Copyright (c) 2014 Carhuatanta, Shea, Herman and Jankord. This is an open-access article distributed under the terms of the Creative Commons Attribution License (CC BY). The use, distribution or reproduction in other forums is permitted, provided the original author(s) or licensor are credited and that the original publication in this journal is cited, in accordance with accepted academic practice. No use, distribution or reproduction is permitted which does not comply with these terms. 